\title{
APORTACIÓN AL DEBATE SOBRE LAS REORIENTACIONES CIENTÍFICAS EN LAS RELACIONES NATURALEZA-SOCIEDAD
}

POR

JAUME MATEU I GIRAL

\section{Introducción}

En la historia de la Geografía la discusión sobre las relaciones entre naturaleza y sociedad se pierde en la noche de los tiempos. Este debate ha producido teorías y ha promovido, más o menos implicitamente, una parte importante de las investigaciones geográficas. Incluso no resultaría arriesgado mantener la postura de que los debates han impregnado e incentivado la evolución del pensamiento geográfico.

De cualquier modo que los geógrafos hayan caracterizado a la Geografía: ciencia de los paisajes, ciencia de los medios naturales para una ecología humana, ciencia de las formas de la diferenciación espacial, ciencia del espacio, geoanálisis, rama del conocimiento concerniente al estudio de los fenómenos naturales y humanos en el espacio accesible a los hombres, etc., se advierte el deseo de estudiar las relaciones entre los hechos humanos (que estudian específicamente las ciencias humanas, sociales o económicas) y los datos naturales (que son objeto de las ciencias de la materia y de la vida).

Jaume Mateu i Giral. Departamento de Geografia Física y Análisis Geográfico Regional. Universitad de Barcelona. 
Esta relación entre naturaleza y sociedad, que está en la base de la organización del saber, tiene plena actualidad ya que los geógrafos se sienten afectados por los síntomas de la crisis de la sociedad y se plantean los problemas en términos de espacio-tiempo en el estudio de las relaciones entre sociedad y naturaleza.

\section{El discurso posibilista de origen vidaliano}

En opinión de J. Crespo Redondo (1992), «sobre las relaciones entre sociedad y naturaleza encontramos dentro de la Geografía un discurso posibilista de origen vidaliano que, en opinión de V. Berdoulay, conserva su interés y es susceptible de actualización y una serie de discursos ambientalistas, algunos de ellos de raíces muy remotas, vinculados al determinismo, que convendría rechazar y evitar».

Prosigue el mismo autor con la siguiente afirmación procedente de V. Berdoulay (1988): «Efectivamente la noción vidaliana de género de vida es un auténtico sistema socioecológico que contempla al mismo tiempo la transformación del medio físico por los hombres y la adaptación de éstos a la naturaleza. Tanto la naturaleza como la sociedad tienen atribuidos papeles activos. Es cierto que el posibilismo de Vidal de la Blache surgió en el contexto de la Geografía regional tradicional, pero ello no impide utilizar su teoría sobre las relaciones entre naturaleza y sociedad y aplicarla en explicaciones geográficas que descansen en otras concepciones del espacio. El discurso posibilista vidaliano es susceptible de actualización gracias a las aportaciones que el constructivismo, la Teoría de Sistemas, la Antropología y cierto pensamiento filosófico han hecho a la interpretación de las relaciones entre la naturaleza y sociedad. Se trata de corrientes convergentes que permiten mejorar la conceptualización de las interacciones. Quizás a partir de todo ello podremos los profesores enfocar el estudio de los problemas ambientales sin distorsionar la lógica de la Geografía».

Según V. Berdoulay la aportación fundamental de P. Vidal de la Blache y sus discípulos a la ciencia geográfica ha sido «aportar el medio para superar la alternativa, o enfrentamiento, positivista entre un determinismo basado en la naturaleza y otro basado en la sociedad, es decir entre el determinismo ambiental y lo que nosotros hemos llamado posibilismo radical».

$$
-34-
$$


La orientación neokantiana proporcionó a los posibilistas la alternativa metodológica que permitió evitar el determinismo ambiental y el determinismo social. La tesis central de Kant era que «el espíritu humano toma parte activa en el conocimiento» y ello permitía desligarse al hombre del yugo de la naturaleza y, por derivación, romper la eterna contradicción y división entre Geografía física y Geografía humana.

A partir de aquí, intentaron saber cómo se armonizaban los datos de la experiencia y la capacidad del espíritu humano para conceptualizarlos.

Los neokantianos afirmaban que el conocimiento, incluso empírico, no es una simple reproducción de los objetos, sino su elaboración por parte del espíritu humano. Según V. Berdoulay, «esta tendencia idealista, no era exacerbada en el sentido que los idealistas, en su mayor parte, no negaban la realidad de las cosas (...) y consideraban que era la experiencia la que otorgaba al conocimiento todo este contenido. El resultado fue que los objetos de conocimiento no se oponían al sujeto, en la misma medida en que ellos mismos eran, en parte, un producto del sujeto».

Los vidalianos, pues, sacaron partido de esta orientación neokantiana, en el estudio de la relaciones hombre-medio. Se resistían a oponer estos dos términos, aunque no consideraban pasivo a ninguno de ellos. En definitiva, se veía en el hombre un colaborador de la naturaleza, en la medida en que él no alcanzaba sus designios más que por medio de ella.

Los vidalianos subrayaron, también, que la acción creativa del hombre se revela cuando éste pone en relación diversos elementos de la naturaleza, hasta entonces dispersos. Esta acción provoca, pues, la aparición de nuevas organizaciones en la superficie terrestre, firmes testimonios de su poder para otorgar orden a los datos empíricos de la experiencia.

$\mathrm{Al}$ parecer de $\mathrm{V}$. Berdoulay, este apoyo neokantiano a la elaboración del posibilismo vidaliano le aportó un enfoque científico que evita los callejones sin salida a que conduce el positivismo. La filosofía convencionalista permitió a los vidalianos recurrir a la utilización de «principios» para conducir sus investigaciones. En la base de estos principios se hallaba la idea de «unidad terrestre» ya pregonada por Humboldt en su Cosmos. A partir de ahí Vidal de la Blache se apoyó

$$
-35-
$$


en la forma de contingencia que constituye la «individualidad», es decir, el resultado relativamente estable de la intervención de series causales independientes. En aquellos momentos tuvo que hacerlo así ya que la «unidad terrestre» estaba fuera de control de la experiencia del hombre de aquellos días. No obstante, dados los modernos recursos para el conocimiento y los avances técnicos, esta experiencia es más posible para el científico actual. En aquellos momentos la atención de los vidalianos se centró en revelar las series causales de fenómenos, más que en la búsqueda de leyes generales como hacían los positivistas.

Desde una perspectiva actual pensamos que buena parte de los seguidores de P. Vidal de la Blache no tuvieron en cuenta estas aportaciones del posibilismo vidaliano, estereotipándolo en «probabilismo» o en posibilismo radical. En definitiva se abandonó el sentido histórico del posibilismo vidaliano $\mathrm{y}$, en consecuencia, éste se estancó al abandonar sus bases epistemológicas. Estamos en la creencia de que, efectivamente, P. Vidal de la Blache situó en la cima de la jerarquía de las labores geográficas universitarias a la monografía regional, pero también pensamos que muchos de sus seguidores casi la fosilizaron en esta situación y de ahí se derivó un perceptible bloqueo conceptual respecto al análisis regional. De lo contrario no puede entenderse el largo olvido de los Principios de Geografía humana de Vidal de la Blache (1921). No obstante ciertos geógrafos (A. Demangeon en las cuestiones políticas o económicas; J. Dresch, pionero en la Geografía de los capitales) o escuelas profundizaron en diversos aspectos y, con la aparición de nuevas herramientas conceptuales y metodológicas, constituyen los gérmenes del mejoramiento en la estructuración de la teoría general vidaliana. Analicemos estas aportaciones conceptuales y metodológicas.

\section{Las convergencias conceptuales}

El estructuralismo de J. Piaget.-Según V. Berdoulay las convergencias más notables, con el posibilismo vidaliano, proceden, en general, de las investigaciones llamadas estructuralistas. Entre ellas, la más globarizadora es probablemente la de J. Piaget (1896-1980) y su escuela. Debemos precisar que J. Piaget propone entender el estructu-

$$
-36-
$$


ralismo como método y no como «doctrina» ya que, según E. Severino (1987), este término confiere rigidez al movimiento de la investigación. El estructuralismo tiene sus raíces en las tesis organicistas de H. Spencer. Para los organicistas «el todo se organiza en un complejo interaccionante» al igual que el símil de un organismo. J. Piaget se interesó muy pronto por la importancia que tenía el medio en el desarrollo de los organismos y afirmó que la adaptación del sujeto al medio se realiza a través de un doble proceso: asimilación-acomodación y equilibrio-desequilibrio. Según el pensador suizo se llega al nivel de equilibrio cuando el sujeto asimila en sus estructuras internas (cognitivas y biológicas) los datos de la experiencia. Es así como, la inteligencia del sujeto, facilita su adaptación al medio exterior. Por tanto, en opinión de Piaget, la inteligencia ya no es solamente una facultad que se posee aleatoriamente desde el nacimiento sino que debe construirse en etapas sucesivas. El aprendizaje, entonces, sería mucho más que el almacenamiento de información: sería una construcción activa del significado. Esta teoría recibe el nombre de constructivista y, al parecer de numerosos expertos, es la que ofrece, en estos momentos, una mejor interpretación del proceso enseñanzaaprendizaje.

El Piaget constructivista intenta, pues, establecer la primacía en la relación organismo-medio abandonando todo determinismo ambientalista o adaptación mecánica de uno a otro.

Según V. Berdoulay, «en términos de relaciones hombre-medio, se pude apreciar que las preocupaciones de Piaget se acercan a las de Vidal de la Blache. Nuevos procesos y equilibrios se forman después de una interacción entre el hombre y su medio ambiente, que implican una reestructuración de las organizaciones propias de cada una de las entidades. Además, los dos autores atribuyen al hombre la primacía en esta interacción en el sentido de que es en él donde hay que buscar el origen de las variaciones de comportamiento. El medio no juega en este sentido el papel de causa iniciadora de novedad. Este es más bien un problema que debe resolver el sujeto mismo, así como el del material para solucionarlo, conservando al mismo tiempo su dinámica propia».

La convergencia general (a pesar del lenguaje utilizado y de la propia concepción del sujeto) entre posibilismo vidaliano y constructivismo piagetiano, las preocupaciones comunes en las relaciones 
hombre-naturaleza son tan similares que sorprende el hecho de su escaso tratamiento por parte de los geógrafos. No obstante, los modelos curriculares geográficos de la reforma de la enseñanza secundaria hacen referencia explícita a la concepcción constructivista del aprendizaje. En el Libro Blanco para la Reforma del Sistema Educativo se indica que esta concepción constructivista tiene en cuenta los conceptos previos del alumno, que «construye» sus conocimientos a partir de sus experiencias; una concepción que busca el cambio conceptual, que entiende la enseñanza como cambio y evolución paulatina. Todo ello basado en una metodología problemática, así como en la especial atención a la adquisición de actitudes y cambio en el papel de profesor, quien de transmisor pasa a ser coordinador del trabajo de sus alumnos, dando importancia al trabajo en grupo y a la interacción.

Sin duda el apoyo constructivista en la conceptualización de las relaciones hombre-medio, puede constituir una base de progreso para la Geografía, por ejemplo «colaborando en la valoración de ciertos mecanismos de adaptación, ensayos o estrategias que caracterizan la actividad humana en relación con su medio».

El estructuralismo de C. Lévy-Strauss.-El propio L. von Bertalanffy escribió en el prefacio a la edición revisada de la Teoría General de los Sistemas que no había previsto «con todo, que la Teoría General de los Sistemas habría de desempeñar un importante papel en las orientaciones modernas de la Geografía, o de ser paralela al estructuralismo francés (por ejemplo, J. Piaget o C. Lévy-Strauss) y ejercer considerable influencia sobre el funcionalismo sociológico estadounidense (1976, p. XIII).

El estructuralismo de C. Lévy-Strauss y su escuela no insiste tanto en los mecanismos psicológicos de desarrollo como en la dimensión cultural del hombre. El estructuralismo cultural se halla definido en las obras de este autor. Esta orientación intenta evitar un determinismo cultural unilateral e inmutable. La filosofía básica de esta escuela se basa en el hecho de que las transformaciones observadas en el tiempo, dan testimonio de la creatividad humana y de su relativa independencia respecto al medio. El material que elabora una cultura, es aquel del que dispone dada su localización, y el que debe tener en cuenta en sus empresas. Para C. Lévy-Strauss las civilizaciones combinan elementos básicos comunes a toda la humanidad. De-

$$
-38-
$$


trás de la diversidad de culturas existe una unidad psíquica de la humanidad.

El posibilismo vidaliano aparece de nuevo claramente cuando se observa que en la interacción hombre-medio, la primacía se otorga al poder iniciador y creador del elemento humano. Se precisa, por otro lado, que este poder está limitado por las características propias del espíritu humano.

Esta versión del estructuralismo, señala V. Berdoulay, valora el sistema de codificaciones propio de las sociedades humanas, pero, al mismo tiempo, señala su pertenencia al mundo natural. Toda adaptación es un proceso complejo, en el que se ajustan los procedimientos del pensamiento humano y las características del medio.

Al igual que en el posibilismo de Vidal de la Blache, el hombre forma parte indisociable de la naturaleza, gozando al mismo tiempo de una amplia autonomía cultural.

El estructuralismo marxista o fenomenológico: contexto científico y solidez del existencialismo.-Su punto de partida debe de buscarse en una serie de acontecimientos que afectaron, a lo largo del sexto y séptimo decenio, al conjunto de relaciones internacionales: el final de la guerra fría, proceso descolonizador, el síndrome de la crisis del Vietnam. En definitiva la fuerte crisis del sistema de dominación occidental. Todo ello permitió, según H. Capel (1981), «el desarrollo del pensamiento marxista con mayores dosis de libertad, una nueva compresión hacia los países dependientes, la puesta en cuestión de las potencias imperiales y la quiebra de confianza en muchos enfoques dominantes hasta entonces».

Paralelamente aumentaron los conflictos en el seno de las sociedades capitalistas. Se consideraba inaceptable el desfase entre el desarrollo tecnológico y su capacidad productiva y las condiciones de producción y el reparto de beneficios. Surgen así fuertes protestas por las condiciones enajenantes del trabajo y de la vida, por el deterioro de las condiciones de vida urbanas y por la desaparición de la biosfera como resultado del desarrollo del modelo capitalista.

En los congresos internacionales sobre la historia de la Ciencia aparecen conflictos latentes entre la racionalidad de la ciencia moderna y los valores de la vida humana y se toma conciencia de la naturaleza esencialmente social del proceso de investigación científica.

En los años sesenta aparecen movimientos y corrientes críticas 
en las Ciencias Sociales (CC. SS.) que se autodenominan radicales, por su pretensión de cambio radical en la concepción social. Sociólogos y economistas buscan en las fuentes marxistas los mecanismos causantes del atraso y del crecimiento económico.

La aparición del libro de P. Baran (1959) sobre economía política supone un cambio en los estudios económicos sobre el desarrollo. De forma explícita se acepta la teoría marxista como punta de lanza radical en forma de economía crítica. Se ataca a las Ciencias Sociales por ser «demasiado limitadas, especializadas, compartimentadas, irrelevantes y sometidas a las técnicas e intereses del «status quo».

La filosofía básica parte de la teoría crítica elaborada por la Escuela de Frankfurt. Se pretende desarrollar una política económica radical para desmitificar la ortodoxa y desenmascarar, con ello, el papel conservador y reaccionario de las CC. SS.

En España, aunque más tardiamente, aparecen movimientos críticos acorde con los americanos, franceses o italianos.

En los trabajos de la Escuela de Frankfurt aparecen profundas reflexiones sobre variadísimos temas con intención de superar la fragmentación del conocimiento científico. En la Teoría crítica se trata de integrar el conocimiento puro (objetivo esencial de la Teoría tradicional) con la acción; se demostraba que los científicos e investigadores están influidos por sus propios valores (problema de la ideología), se introduce la dimensión histórica en los estudios y se reconoce que la imaginación estética y la fantasía son necesarias para entender la realidad social. Al mismo tiempo, según H. Capel, se rechazaban los planteamientos exclusivamente teóricos y abstractos, así como el fetichismo en la lógica y las matemáticas, cuya significación social se considera escasa. Todo ello comporta una actitud claramente antipositivista de la Escuela de Frankfurt que se acentuó a partir de 1961, con la presentación de una ponencia de T. Adorno sobre Sociología e investigación empírica, en el Congreso de la Sociedad alemana de Sociología, que representa una crítica frontal a la sociología empírica. Adorno propugna una sociología integrada que se pregunte por las conexiones esenciales y no subdividida en disciplinas cada vez más especializadas. En ella lo esencial es elaborar teorías sociales y partir de ellas para el análisis de la realidad ya que los datos averiguados por la investigación empírica «no reflejan fielmente las relaciones sociales subyacentes, sino que al mismo 
tiempo componen el velo con que aquéllas, de manera necesaria desde luego, se embozan».

T. Adorno, en respuesta a la exposición de K. Popper en el citado congreso, proclamó como algo innegable que «el ideal epistemológico de la elegante explicación matemática, unánimemente y máximamente sencilla, fracasa allí donde el objeto mismo, la sociedad no es unánime ni es sencilla». Con ello, según $\mathrm{H}$. Capel, se rechazaban de forma explícita varios de los postulados básicos del positivismo lógico y su intento por establecer una ciencia unitaria que pudiera examinar de forma «objetiva y neutra la sociedad».

La noción «ideología» surgía una y otra vez, y, también, repetidamente se aparejaba a la «objetividad científica», llegando a la conclusión de que los investigadores en CC. SS. no pueden ser objetivos ya que en palabras de J. Muguerza (1975) «la ciencia no es sólo un lenguaje bien hecho, sino una compleja actividad humana, en cuanto tal inmersa en la infinita complejidad de las demás actividades de los hombres».

La consecuencia es que el criterio de demarcación entre lo científico y lo no científico es menos claro de lo que pretendían los positivistas $\mathrm{y}$, por tanto, vuelve a plantearse otra vez el problema de la explicación científica, incluso en las ciencias naturales, ya que la existencia de lo aleatorio y lo indeterminado dificultan las predicciones exactas.

Por todo ello se va imponiendo lo que J. Muguerza llama un «sano pluralismo» en materia de investigaciones científicas, pluralismo al que desde una perspectiva geográfica hemos aludido en ocasiones. Desde lugares y disciplinas diversas se toma conciencia de lo que P.M. Sweezy (1972) llamó «el abismo verdaderamente asombroso entre los triviales problemas planteados y las refinadas técnicas utilizadas para resolverlos» y se pone en duda lo que ha dado en llamarse el mito de la metodología y el hecho de que la explicación científica basada en el mundo de las ciencias naturales pueda aplicarse a las disciplinas sociales.

Es en este contexto que la fenomenología y el existencialismo adquieren un especial relieve. La valoración que hacen de la conciencia y de la experiencia personal aparecen como vías alternativas frente a las abstracciones del cientifismo positivista. Los fenomenólogos, basándose en el pensamiento de H. Husserl (1859-1938), sostienen la 
tesis de que el mundo de la vida es «un reino de evidencias originales», algo subjetivo y muy distinto, por consiguiente, del mundo objetivo y abstracto de la ciencia. Para H. Husserl, en el abismo abierto entre ciencia moderna, cada vez más abstracta y técnica, y mundo vivido por el hombre deberían buscarse las claves fundamentales que nos aproximan a la crisis del pensamiento occidental. La idea de $\mathrm{H}$. Husserl y la Escuela fenomenológica la describe A. Buttimer recogiendo una frase de E. Cassirer (1953): «nuestra riqueza de datos no supone necesariamente una riqueza de pensamientos».

Después de la Segunda Guerra Mundial el existencialismo abandona un tanto la línea fenomenológica y se preocupa «por la relación hombre-mundo, de las posibilidades y limitaciones que éste ofrece a los objetivos y aspiraciones del hombre». Centra su atención en la existencia humana y en su temporalidad y, por tanto, en su historicidad. M. Heidegger (1889-1978), K. Jaspers (1883-1963), J.P. Sartre (1905-1980) reflexionan sobre el sentido de la vida, el comportamiento humano, su existencia y conceden a la ciencia un papel menos determinante que los fenomenólogos.

La fenomenología y el existencialismo impulsaron, en las Ciencias Sociales, el enfoque directo, vivencial, no abstracto, valorando la observación participante del investigador; difundieron una preocupación constante por la vida cotidiana, recuperando el campo de la experiencia personal, preparando el camino hacia un nuevo ideal científico en CC. SS., socavando los fundamentos de la Geografía cuantitativa.

El surgimiento $y$ fortalecimiento de las geografias radicales.-Bajo estas orientaciones va apareciendo la Geografía radical que en sus orígenes tiene unos enfoques claramente procedentes de la percepción y del comportamiento, ahora tratados de una forma consciente. Las perspectivas de la Psicología comprensiva se introducen así en los estudios geográficos. Se ponen de manifiesto la influencia de las actividades humanas. Weber, Lösch y el propio Christaller son criticados y sus modelos «optimizadores» puestos en evidencia frente a los modelos «satisfizadores» que ponen el énfasis en la diversidad de motivaciones que influyen en la toma de decisiones del hombre (no únicamente decisiones económicas, consumistas y racionales) como puso de manifiesto H.H. Simon (1957).

J. Wolpert aplica, en el campo de la Geografía, las ideas de 
H. H. Simon llegando a la conclusión que el comportamiento real del hombre se ajusta más al principio «satisfizador» que al «optimizador», propone un enfoque behaviorista (procesos de percepción y decisión personales) para entender correctamente este fenómeno. De ahí se sigue el gran interés por la Geografía del comportamiento, percepción, decisión, aprendizaje para adecuarse a nuevas situaciones..., con lo cual la Geografía tiende su análisis hacia la Psicología.

Ahora interesan temas como la percepción de catástrofes naturales, las condiciones climáticas o físicas del medio, actitudes ante el medio, evaluación de los recursos, percepción del paisaje natural y urbano, imágenes espaciales y mapas mentales, conciencia territorial y regional y, en definitiva, el espacio vivido tal como es vivido en la realidad. Ello enlaza con los enfoques fenomenológicos y existenciales.

En los años 70 la toma de conciencia sobre nuevos problemas reales (problemas ecológicos, segregación social en las ciudades, descubrimiento de la injusticias dentro y fuera de las sociedades occidentales, guerra del Vietnam y, en EE.UU., la conciencia de pertenecer a un país imperialista y explotador), hace aparecer un movimiento crítico radical cuyo órgano de difusión fue la revista Antipode que aspira a situarse en las antípodas de la Geografía que se practica en estos momentos. Una Geografía comprometida que contribuya a los cambios revolucionarios que la sociedad necesita. $R$. Peet, alma de la revista, indica que la Geografía se ha alejado del conocimiento del mundo como es, sobre todo en su vertiente social. En esta idea inciden prestigiosos geógrafos como F. Donaldson o R. Morrill que, en el primer número de la revista (1969), esgrimen: «... la pobreza, la injusticia, la discriminación, el hombre, la contaminación o el amontonamiento humano han sido pasados por alto». Aparecen así temas inesperados (pobreza, hambre, violencia, ghettos urbanos) que incorporan explícitamente la dimensión espacial. El marxismo se revela como soporte teórico adecuado a la nueva temática que aparecen en escena. D. Harvey piensa que ciertos aspectos del positivismo, materialismo y fenomenología confluyen con el marxismo.

La Geografía radical va haciéndose cada vez más marxista aunque con notables dificultades. Por ejemplo Y. Lacoste, en la presentación (1976) del $\mathrm{n}^{0} 1$ de la revista Hérodote, anunció que la Geografía es la Ciencia Social en la que el análisis marxista tiene más dificulta- 
des para desarrollarse, sobre todo por la pretendida ausencia de una reflexión marxista sobre el espacio, debido a que Marx concedió importancia determinante a las relaciones de producción y a la lucha de clases.

La reacción antipositivista del marxismo también inspiró a la otra gran corriente de la Geografía radical, la Geografía humanista que pone énfasis en los significados, valores, objetivos y propósitos de las acciones humanas. La Geografía humanista sustituye el enfoque objetivo, abstracto, mecanicista y determinista por el enfoque subjetivo, cognitivo, globalizador y comprensivo.

Un destacado Geógrafo humanista, Tuan Yi-Fu (1976) manifestaba en la revista Annals (vol. 66, $\mathrm{n}^{\circ}$ 2) de la A.A.G. que «el objetivo básico es comprender el hombre y su condición»y, por tanto, puede interpretarse que la Geografía humanista se distancia de las ciencias de la Tierra volviendo al típico dualismo historicista. Proceso lógico teniendo en cuenta que la dimensión subjetiva y experiencial (procedente de la Geografía de la percepción y del comportamiento) es básica en el análisis de la Geografía humanista.

Para los geógrafos humanistas el hombre no es solamente un ser económico sino un ser social con necesidades de relación y con unos valores culturales y sociales. Los temas de análisis geográfico girarán alrededor de los valores, el mundo de la vida, el espacio vivido y se critican abiertamente el espacialismo y el economicismo de la Geografía cuantitativa. Las críticas se extienden con frecuencia al marxismo, a pesar de las líneas coincidentes que ofrecen la fenomenología y el existencialismo.

A. Buttimer (1976) en la revista ya citada, afirmaba que el «homo economicus» debe sustituirse por el «homo sapiens» más orientado hacia sus propias necesidades para sobrevivir y crecer, en diálogo con la naturaleza, con el espacio y con el tiempo».

El concepto de espacio (abstracto) es substituido por el de lugar (concreto y experiencial). El interés se desplaza hacia el «mundo vivido», un mundo que ha de ser aprehendido y experimentado en su totalidad, de forma holista, sin simplificaciones ni abstracciones que deformen la realidad. El objetivo del geógrafo es la comprensión, el contacto, el compromiso, la relación, la autenticidad de los hechos. Es decir, la observación participante o el trabajo de campo experiencial. El método, por tanto, es decididamente inductivo y vuelven a in-

$$
-44-
$$


teresar las reglas del trabajo de campo de la Geografía regional convergiendo con el posibilismo vidaliano.

Los geógrafos humanistas estudian el espacio egocéntrico, organizado a escalas diversas -desde la casa al espacio mundial-. La estructura del espacio existencial es lo tratan de aprehender. Pero el espacio está cargado de historia, movilidad y contingencia y ello se analiza en sus estudios. Vuelve a interesar la Geografía histórica desde una perspectiva crítica con el pasado y, en consecuencia, se han revaluado los estudios regionales franceses.

A. Buttimer, figura destacada de esta corriente, ha valorado la filosofía geográfica de P. Vidal de la Blache y ha relacionado sus ideas con las de la Escuela fenomenológica de H. Husserl. Algo parecido ocurre con otros destacados geógrafos humanistas.

Según A. García Ballesteros (1992), la Geografía humanística aporta la reformulación de ciertos conceptos, el recurso a técnicas de trabajo que suponen un mayor contacto con los lugares y la vida cotidiana, aportaciones que, sin duda, contribuyen a enriquecer la Geografía en la exaltación de la individualización, del tiempo, del espacio y de la propia historia. Asimismo cabría añadir que esta tendencia ayuda a superar el posibilismo radical en que habían caído determinados círculos marxistas como consecuencia de concebir exclusivamente el espacio como producto social, conducente a una teoría científica de la evolución social, basada en un fuerte grado de determinación. Ahora aparecen de nuevo viejas polémicas que se produjeron a propósito de la Geografía regional, reabriéndose, creemos, un debate que jamás debió cerrarse.

Las intransigencias de tiempos pasados no deben reavivarse ni obstaculizar, en el presente, posibles desarrollos que pueden llegar a ser fecundos en el ámbito geográfico. La premisa no debe ser positivismo o historicismo o ideal de las ciencias matemáticas frente a ideal de conocimiento histórico o, si cabe, empirismo frente a idealismo. Antes bien, entendemos que debe prodigar la comprensión y coexistencia de ambos dogmas, ya que, con toda seguridad, no son exclusivos ni excluyentes tal como opina el gran pensador E. Cassirer (1979).

El modelo basado en el aparato científico-técnico.-Al parecer de V. Berdoulay (1983) las recientes reflexiones acerca de la técnica (sus relaciones con la creatividad humana, con la adaptación al medio am- 
biente, con la transformación de la naturaleza y con la sociedad) tienen interés en la actualización del posibilismo vidaliano. La transformación de la naturaleza por parte de la ciencia y de la técnica se convirtió durante la Revolución Industrial en una preocupación social generalizada como muestran las obras de grandes pensadores del momento como Dickens o Zola. De esta preocupación social parte la idea de determinismo tecnológico, analizada por L. Mumford (1978).

Las consideraciones geográficas sobre la técnica, según Mumford, se centran en dos conceptos: el paisaje y el modo de vida. Tanto uno como otro representan dos tipos de estructuracion de fenómenos tanto sociales como naturales, y se basan en una visión histórica de las relaciones hombre-medio.

Estas reflexiones pueden reforzar la postura posibilista. Según V. Berdoulay «la problemática posibilista no pude hacer más que facilitar el que se tenga en cuenta la técnica, en la medida en que ésta está integrada en el proceso de construcción de la realidad, más que considerarla como una entidad aparte, como una simple herramienta cuya utilización estaría sometida a juicios morales».

También el posibilismo vidaliano puede ayudar a superar los ecos del temor que la tecnología llevaría a la sociedad a una «economía destructiva» (Raubwirtschaft) de la Tierra, ya manifestados por J. Brunhes (1912) en su Geografía humana. Influenciado, sin duda, por las tesis de su hermano B. Brunhes (1908), físico interesado en las cuestiones de la energía y en cómo la forma en que podría utilizarla el hombre podría hipotecar su futuro.

El espectacular fortalecimiento e influencia de la economía liberal en las últimas décadas ha obstaculizado el desarrollo de esta teoría, aunque en la actualidad a través del concepto "desarrollo sostenible» vuelve a plantear interesantes debates: la finalidad del aparato científico-técnico, su fraccionamiento, sus relaciones con las ideologías y el poder, las soluciones que aporta a la nueva conflictividad del Planeta, etc, son consideraciones interesantes y novedosas (E. Severino, 1991).

Teniendo en cuenta las recientes investigaciones, descritas en los apartados anteriores el posibilismo vidaliano como Teoría general de las relaciones hombre-medio, merece ser salvado del olvido en el cual ha quedado progresivamente postergado, pero también es evidente que se impone la necesidad de poner al día su metodología. Avance 
metodológico que quedó cortado según W. Davies (1966) por el rechazo frontal al determinismo por parte de la Escuela posibilista.

Un instrumento eficaz para actualizar el discurso posibilista procede del análisis sistémico (en sentido amplio), sobre todo en base a los recursos conceptuales y metodológicos que proporciona. Estos se adaptan bien, según V. Berdoulay, a la reflexión sobre las relaciones hombre-medio.

\section{El análisis sistémico.}

L. von Bertalanffy observó que para determinar las leyes que rigen la vida de un organismo era necesario estudiarlas como un sistema, como un todo que funciona debido a la interdependencia de sus partes. También se dió cuenta que esta idea era aplicable no sólo a los sistemas biológicos sino que podía trasladarse a los que no lo eran, y que estos sistemas tenían muchas características comunes en una serie de ciencias. Fue, pues, posible desarrollar una Teoría General de los Sistemas que proporcionara una estrucutura y un procedimiento analítico válido para todas las ciencias. Un sistema general es, según H. Jensen (1992), una generalización de orden superior de gran cantidad de sistemas que cada ciencia había reconocido por su lado.

A partir de la II Guerra Mundial, el aumento progresivo de investigaciones interdisciplinarias y el desarrollo de métodos cuantitativos y de la informática, prepararon a la ciencia para una Teoría General de los Sistemas.

Todos los sistemas reales son complejos indivisibles compuestos por un sinfín de variables. A partir de un sistema real podemos formar distintos sistemas abstractos, en base al establecimiento de modelos que permitan llevar a cabo una forma determinada de análisis.

Los sistemas reales son abiertos, pero en nuestros estudios sobre sistemas sólo podemos tener en cuenta un número determinado de elementos dentro del sistema y las relaciones recíprocas que se dan entre ellos. Por tanto, un sistema al ser analizado se convierte en cerrado, puesto que lo abstraemos a un modelo forzosamente limitado, reducido a los elementos y conexiones previamente decididos, en función de nuestros intereses investigadores. 
En definitiva, el análisis de un sistema sólo es posible después de haberlo delimitado. En la metodología geográfica es comporta considerables problemas a la hora de escoger o delimitar los elementos. Identificando el grupo de elementos que describen mejor el sistema real, podemos construir un sistema abstracto para modelar una situación real.

Según Holt Jensen (1992) todo sistema puede estudiarse en base a tres aspectos fundamentales: estructura, función y desarrollo. La estructura es la suma de elementos y las relaciones o conexiones entre ellos. La función se refiere a los flujos (relaciones de intercambios) que tienen lugar en las conexiones. El desarrollo representa los cambios que tienen lugar con el tiempo, tanto en la estructura como en la función. En el análisis de los elementos aparecen dos problemas básicos a resolver: decidir la escala e identificar los elementos a utilizar.

El análisis del desarrollo de un sistema se refiere a las influencias que recibe del entorno y que afecta a sus elementos. Estas influencias pueden o no alterar la estructura del sistema, siendo mucho más problemático el estudio de sistemas alterables con el paso del tiempo, que los sistemas estáticos o adaptables.

Los geógrafos se interesan, ante todo, por el estudio de sistemas cuyas variable funcionales más importantes son las condiciones espaciales, tales como la localización, distancia, extensión, densidad, etc. Un sistema en el que dos o más variables, funcionalmente importantes, son espaciales se puede describir como sistema geográfico. Es difícil construir un sistema geográfico dinámico ya que debemos combinar espacio y tiempo en el mismo modelo lo que nos lleva a representaciones tridimensionales, difíciles de analizar y manejar.

Este autor concluye que el análisis de sistemas es todavía una disciplina joven y precisa de un mayor desarrollo para manejar los complejos fenómenos geográficos. En un proyecto de investigación tal no podamos analizar más que unos cuantos factores, pero sin duda el modelo de sistemas, aunque conceptual, nos ayuda en la comprensión de relaciones complejas.

Otras consideraciones se fundamentarán, entre otros trabajos, en los estudios del propio V. Berdoulay (1983), de S. Quesada (1978), M. García Pelayo (1975), R. Méndez y F. Molinero (1991). 
APORTACIÓN AL DEBATE SOBRE LAS REORIENTACIONES CIENTÍFICAS...

Para V. Berdoulay (1983) la Teoría General de Sistemas permite:

- Estimular los intercambios interdisciplinares.

- Encontrar multitud de interacciones hasta hoy desconocidas.

- Proporcionar el abandono de las anteojeras de las especialidades que el positivismo tiene tendencia a fomentar.

- Ayudar a superar ciertos hábitos decimonónicos que supuestamente practican nuestros científicos.

- Descubrir la complejidad sin multiplicar ni complicar los métodos existentes, en aras de la exhaustividad, en el análisis de las variables.

- Buscar la simplicidad y generalidad en la formulación de teorías.

- Ayudar a los geógrafos y a la Geografía a superar el lugar marginal que ha ocupado, durante décadas, respecto a otras disciplinas sociales.

- Adoptar un caríz próximo al posibilismo vidaliano.

Esta aproximación se puede lograr, por ejemplo, a través del concepto "sistema abierto» porque en él se produce una entrada de estímulos (energía) exterior al sistema. Este concepto fue acuñado por L. Von Bertalanffy (1968) que sostenía la teoría de que los sistemas abiertos tienen tendencia a la desintegración y, por tanto, para su sostenimiento son imprescindibles los intercambios de energía con el medio ambiente. Asimismo cree que el sistema abierto se autoorganiza y en el intento de analizar y explicar la autoorganización del sistema se establece la subjetividad y la libertad individual del investigador. Bertalanffy, en el prefacio de su obra, indicaba que «representa un amplio punto de vista que transciende grandemente los problemas y los requerimientos tecnológicos, una reorientación que se ha hecho necesaria en la ciencia en general, en toda la gama de disciplinas que va de la Física y la Biología a las Ciencias Sociales y del comportamiento, y hasta la Filosofía. Con distintos grados de éxito y de exactitud, interviene en varios dominios y anuncia una nueva visión del mundo que tendrá repercusiones considerables» (1968, p. VIII).

Particularmente unido a la concepción de sistema abierto se hallaría el concepto de ecosistema que ha sido usado por los geógrafos como modelo para interpretar la realidad. Por ejemplo D.R. Stoddart 
(1967) valora esta utilización en base a cuatro características esenciales que presentan los ecosistemas:

a) Concepto monista que permite integrar lo orgánico y lo inorgánico. Ello supone para la Geografía, evitar el conflicto entre Geografía humana y física.

b) $\mathrm{Su}$ estructuración ordenada y racionalmente comprensible. Por tanto, por mucho que las estructuras del ecosistema se desarrollen pueden ser investigadas y comprobadas.

c) $\mathrm{Su}$ funcionamiento mediante el intercambio de materia $\mathrm{y}$ energía.

d) Configuran un sistema abierto que tiende hacia una situación de equilibrio y, por tanto, presenta las características de los sistemas generales que utiliza las teorías del análisis de sistemas.

A las cuales, H. Capel (1981) añade que «es capaz de una estructuración matemática precisa y puede ser abordado en términos de cibernética, teoría de información y de la comunicación. Nos hallamos pues, ante esta última característica, en una reformulación positivista del enfoque sistémico aplicado al análisis de los ecosistemas». Aunque, entendemos, cabría precisar que una cuestión es la epistemología de la ciencia (desde esta perspectiva L. von Bertalanffy difería del positivismo o empirísmo lógico por considerar sus planteamientos analíticos anticuados) y otra, bien diferente, la actitud científica (que L. von Bertalanffy compartía con el positivismo lógico).

Para S. Quesada (1978) la Teoría General de los Sistemas:

- Propicia un marco general para explicar el funcionamiento de los sistemas en base al uso de múltiples variables.

- Ofrece posibilidades técnicas a la Geografía para establecer postulados teóricos y un mayor grado de relación con otras ciencias.

- Ayuda a comprender el cómo y el porqué de determinado funcionamiento de un sistema natural y/o humano en sus dimensiones espaciales y temporales (conocer la estructura del sistema), además de conocer los procesos de cambio.

- Permite pasar de «modelos estáticos» explicativos de las regularidades a «modelos dinámicos» $\mathrm{y}$, por ende, conduce a la predicción e intervención en los procesos naturales y sociales.

$$
-50-
$$


- Manifiesta la posibilidad de conocer las diferencias, discontinuidades, relaciones y tendencias en la dinámica del territorio y de la sociedad, salvando la distancia existente entre teoría y práctica mediante el estudio dinámico de procesos.

- Propicia la formulación de leyes generalizables en los aspectos físicos, biológicos y geográficos.

Los problemas clave de la Geografía, según diversos autores, pueden abordarse a partir de la Teoría General de los Sistemas. En este sentido una de las propuestas iniciales fue desarrollada por E. Ackerman en 1963 al decir que el problema clave de la Geografía «es nada menos que la comprensión de un enorme sistema de interacción que comprende toda la humanidad y a su medio ambiente natural sobre la superficie terrestre». En los años 60, este autor insistía en la idea de que la técnica de sistemas jugaría un papel cada vez mayor a la hora de hacer frente a la crisis social y económica que traía consigo el desarrollo tecnológico.

R. Méndez y F. Molinero afirman que la «revisión teórica de los estudios regionales implica necesariamente una paralela transformación metodológica que supere la mera yuxtaposición de elementos físicos y humanos. Desde las afirmaciones precedentes, la consideración de cualquier aspecto sólo cobra verdadero significado en relación con su funcionalidad dentro del sistema espacial en que se integra, es decir, únicamente a partir de un contexto global cabe situar el lugar y la importancia otorgadas a cada variable (...). En cada región, pues, habrá de darse prioridad al análisis de sus estructuras básicas o dominantes, las que justifican lo esencial de su organización y dinamismo, dejando a un segundo plano aquellas otras subsidiarias que cuentan con menor valor explicativo (...). Respecto al esquema metodológico a aplicar, y frente a la anterior yuxtaposición de contenidos, la comprensión del hecho regional parte de la consideración de aquellos factores internos y externos implicados en cada caso, para analizar después los procesos a que han dado lugar y las estructuras territoriales, resultantes (...). Así según M. Santos (1990) forma, función, proceso y estructura resultan ser las categorías analíticas básicas para fundamentar el estudio de las diversas unidades regionales».

Al parecer de V. Berdoulay, el posibilismo ha alimentado las

$$
-51-
$$


grandes líneas de una verdadera Teoría general de las relaciones hombre-naturaleza. Y esa teoría es la más apta para:

- Orientar de manera distinta las investigaciones contemporáneas en Ciencias Sociales.

- Facilitar la integración de las contribuciones científicas recientes.

- Facilitar el análisis integrado en el estudio de las relaciones hombre-naturaleza.

- Superar una polarización extrema entre Geografía humana y Geografía física.

Por su parte, R. Méndez y F. Molinero (1991) argumentan: «Aun cuando en el momento presente una utilización estricta del análisis sistémico aplicado a la Geografía regional del mundo no resulte aún posible ante la persistencia de problemas teóricos y de información no resueltos, el empleo de algunos de sus conceptos básicos como hipótesis interpretativas de carácter general y la insistencia sobre las interrelaciones espaciales, frente a la simple descripción analítica, puede ofrecer una alternativa coherente dentro de la necesaria revisión crítica a la que se enfrentan los estudios regionales en la actualidad».

Los mismos autores afirman que la Teoría General de los Sistemas «en su preocupación por integrar los contenidos de las diversas ciencias mediante el establecimiento de una lógica común a todas ellas, esta permitiendo la decantación de un amplio conjunto de conceptos, técnicas analíticas y de un lenguaje normalizado que, además de contribuir a superar el creciente aislamiento entre las ciencias (lo que Ortega denominó «la miseria del especialismo»: el saber cada vez más de menos») ha estimulado un desarrollo teórico paralelo en diversos campos de investigación al favorecer las transferencias interdisciplinarias».

El establecimiento de nuevas categorías teóricas; una nueva visión que muestre el territorio como un conjunto de elementos interdependientes; una colaboración estrecha con otras disciplinas científicas interesadas en estos temas... serían algunas de las claves que permitirían superar los obstáculos que van surgiendo en el desarrollo de la Teoría General de Sistemas. 
APORTACIÓN AL DEBATE SOBRE LAS REORIENTACIONES CIENTÍFICAS...

Las perspectivas de futuro: La Teoría del Cambio Global.

De hecho, en los últimos años, en numerosos ámbitos científicos se está trabajando en el desarrollo de métodos de investigación y técnicas que logren superar el excesivo especialismo científico. Uno de los programas internacionales más ambiciosos (1988) trata sobre «Las dimensiones sociales de los cambios en el medio ambiente planetario» coordinado por el Consejo Internacional de Ciencias Sociales (CICS), con la participación de la UNESCO y la Universidad de las Naciones Unidas (UNU). Es un programa interdisciplinario en los niveles teórico y metodológico que puede conducir, en palabras de A. Kazangil (1991), «a progresos importantes en las diferentes áreas de las CC. SS., haciendolas más aptas para el análisis y el seguimiento de los problemas globales medio ambientales y contribuyendo también a la puesta en marcha de políticas apropiadas en este campo».

Este nuevo campo de investigación se denomina en general del «Cambio Global». M. Price (1990) afirma que esta expresión se ha defendido predominantemente desde una perspectiva geocéntrica y biocéntrica, por lo que es necesario progresar hacia la creación de una perspectiva sociocéntrica que, según L. Arizpe (1991), «plantea la exigencia de una nueva ciencia global que integre los programas de investigación de las Ciencias Naturales y Sociales». Un primer paso lo ha constituido el programa analizado por H. K. Jacobson (1990) y otros.

Sin embargo contrariamente a lo que pasa en las CC. NN., las CC. SS. no han logrado identificar con precisión los problemas más urgentes del medio ambiente mundial. Ello no debe de extrañarnos «dada la complejidad de las acciones humanas relacionadas con los múltiples procesos del cambio ambiental global». L. Arizpe argumenta que es preciso instaurar una nueva perspectiva global que permita analizar minuciosamente la realidad existente con un criterio distinto al que ha encauzado la investigación en CC. SS. durante el último siglo.

Según esta investigadora existen varias propuestas para articular esta nueva perspectiva global. Burton y Timmerman (1989) han señalado la aparición de un «nuevo paradigma» basado en lo que denominan los sistemas complejos. No obstante, la creación de un nuevo paradigma supondría no sólo crear un programa teórico de investigación sino también reconsiderar algunas de las premisas epistemológicas bá-

$$
-53-
$$


sicas de las CC. SS. tradicionales. Como están vinculadas a principios filosóficos fundamentales, ello significa que nuevas fórmulas filosóficas y éticas deben formar parte del programa para la creación de conocimientos necesarios, que permitan abordar las nuevas áreas de experiencia humana, tales como la ingeniería genética, así como los nuevos límites de la empresa humana, por ejemplo el agotamiento de los recursos no renovables.

La definición del cambio global en CC. NN. abarca dos criterios básicos:

1) Escala: a partir de la segunda mitad de este siglo la humanidad ha tenido la capacidad de traspasar los límites locales para modificar el medio ambiente a escala global.

2) Interacción: el cambio global entraña procesos físicos, quími$\cos$ y biológicos interactivos que regulan el sistema terrestre; los cambios que se estan produciendo en este sistema y el modo en que las acciones del ser humano influyen en ellos.

Si en las CC. NN. los criterios básicos para definir los fenómenos globales son escala e interacción, según L. Arizpe, será fácil crear una imagen paralela en las CC. SS. que sea equivalente. Concluye que «para entender estos fenómenos bastaría con añadir un nuevo criterio a nuestros modelos y establecer las relaciones entre los diferentes niveles». Pero la interpretación de la realidad global no puede realizarse con el actual modelo, que establece fronteras entre disciplinas y prima unas determinadas categorias de análisis. Esta metodología se estableció para un mundo dividido entre pueblos occidentales y no occidentales, fragmentado en naciones-estado y, regulados por esferas institucionales relativamente estables, que ya no existen. Para L. Arizpe el problema consiste en que «muchos de los esquemas epistemológicos y teóricos más influyentes (individualismo metodológico, positivismo lógico, relativismo, desconstruccionismo) crean barreras metodológicas que se oponen al establecimiento de una perspectiva social de los fenómenos globales. Argumenta que como los fenómenos globales no tiene precedentes en la experiencia humana, no sólo carecemos de los métodos para comprenderlos sino que además no tenemos categorías e ideas básicas que nos sirvan para pensar en los mismos. Así, en el cuerpo tradicional del pensamiento teó-

$$
-54-
$$


rico de las CC. SS. hay pocas hipótesis para interpretar estos fenómenos, lo que puede contribuir a explicar por qué parecen tan invisibles desde el punto de vista de las CC. SS.

Afirma que nuestra interpretación del mundo, en las CC. SS., está aún condicionada por los mapas mentales del siglo xIx y ello hace difícil que podamos observar los fenómenos globales de nuestra experiencia cotidiana. L. Arizpe señala la necesidad de establecer investigaciones interdisciplinares y la creación de modelos microsociales «en base a procesos relacionales» en el análisis de un contexto global o macrosocial. R. Balstad (1991) afirma, no osbtante, que las CC. SS. no son neófitas en el campo de la investigación del cambio global y que «aportan una rica y variada tradición de estudios en temas estrechamente relacionados con el debate ambiental contemporáneo». Insiste en la idea que las CC. SS. -debido al decisivo papel de aceleración de la acción humana, los datos, modelos e investigaciones-, contribuirán a reducir la incertidumbre en zonas clave de la investigación de las CC. NN. en lo que se refiere al cambio global. Al propio tiempo, cuando se tenga en cuenta la dimensión humana en estos cálculos, se plantearan nuevas cuestiones, aumentaran las incertidumbres científicas (debido a la esencia analíticamente confusa de la conducta humana, que es objeto de las CC. SS.), se introducirán incrementos exponenciales en la complejidad de la investigación, lo que causará incomodidad en muchos científicos naturalistas y hará que algunos traten de evitar la dimensión humana. Es esencial para los investigadores en CC. SS. y CC. NN. tratar de evitar esta tendencia.

R. Balstad concluye que la mejor respuesta al debate sobre si las CC. SS. y las CC. NN. deben avanzar juntas o cada una por su lado, la proporcionó el historiador inglés T. S. Ashtib en relación a los debates concurrentes de análisis histórico: «es como discutir si hay que andar con la pierna izquierda o con la derecha; las personas con dos piernas han observado que andan mejor con las dos».

En este sentido, investigadores de pretigio internacional como J. W. M. La Rivière, J. B. Robinson o G. C. Gallopin insisten en la creación y el desarrollo de marcos conceptuales y modelos de relación en la investigación de las interacciones entre sistemas humanos y naturales.

Estas nuevas reflexiones epistemológicas deben ser aprovechadas por los geógrafos y por la Geografía. Si ello no es así esta disciplina 
puede verse involucrada, una vez más, en un bloqueo conceptual y metodológico que provoque el desplazamiento del análisis socio-ecológico hacia otras disciplinas en las que se hayan debatido y digerido estas preocupaciones y reflexiones epistemológicas.

\section{BIBLIOGRAFÍA}

Ackerman, E. (1976) Las fronteras de la investigación geográfica, Geocrítica, $\mathrm{n}^{\circ} 3$, Barcelona.

Adorno, J., Popper, K. y otros (1972) La disputa del positivismo en la sociologia alemana, Colección «Teoría y realidad», Grijalbo, Barcelona.

ARIzPE, L. (1991): «El cubo global», en Revista Internacional de Ciencias Sociales (RICS), $\mathrm{n}^{\circ} 130$, p. 628.

Artal, F. y otros (1972) Economía crítica. Una perspectiva catalana, Edic. 62, Barcelona.

Aliberas, J. (1989) Didáctica de las Ciencias. Perspectivas actuales. Eumo editorial, Col.lecció Interseccions, $\mathrm{n}^{\circ} 11$, Barcelona.

BALSTAD, R. (1991) «Las CC. SS. y el desafío del cambio ambiental mundial», en Cambios en el medio ambiente planetario (RICS), Centre Unesco Catalunya, $\mathrm{n}^{\circ} 130$, diciembre, p. 641.

Baran, P. (1959) La economía política del crecimiento, F.C.E., México.

Berdoulay, V., Phipps, M., Poulin, G. (1982) Ambiance, structure et stratégie: «Recherche sur Iappreciation du paysage forestier par les campeurs», Géographie Canadien, 1982, pp. 93-109.

Berdoulay, V. (1983); «Perspectivas actuales del posibilismo: de Vidal de la Blache a la ciencia contemporánea», Geocrítica, $n^{\circ} 47$, Cuadernos críticos de la Geografía humana, Barcelona.

Bertalanffy, L. von (1972) Teoría general de los sistemas, F.C.E., Méjico. Traducción de la edición inglesa, 1968.

Brunhes, B. (1908) La dégradation de l'énergie, Flammarion, Paris.

Burton, I. y Timmerman, P. (1989) «Human dimensions of Global Chance: a Review of Responsabilities and Opportunities», en International Social Science Journal, $\mathrm{n}^{\circ} 121$.

CAPEL, H. (1981) Filosofía y ciencia en la Geografía contemporánea, Barcanova, Barcelona.

CASSIRer, E. (1979) El problema del conocimiento en la fisolofía y en las ciencias modernas, F.C.E., Méjico, 1953. 3a. reimpresión en 4 vols.

CRESPo REDondo, J. (1992) "Geografía y formacion ambiental en la reforma educativa en España. Un análisis crítico» Boletín de la AGE, $\mathrm{n}^{\circ}$ 14, Madrid, pp. 7-21.

Davies, W. (1966) Theory, science and geography, Tikdschrift voor Economische en Sociale Geografie, Utrecht, vol. 57, julio-agosto. Citado por H. Capel, Fisosofía..., ob. cit.

Dubos, R. (1972) Le monde ininterrompu, Denoel, París.

Garcia Ballesteros, A. (1992) Geografía y humanismo, Oikos-Tau, Barcelona. (Contiene un artículo escrito por Anne Buttimer).

Garcia Pelayo, M. (1975) «La Teoría General de Sistemas», Revista de Occidente, 3a. época, $\mathrm{n}^{\circ} 2$, pp. 52-59.

Jacobson, H. K. y Price, M. F. (1990) «A Framework for Research of the Human Dimensions of Global Environmental Change», París, ISCC/UNESCO, series: 3.

Jensen, H. (1992) Geografía. Historia y conceptos, Vicens Vives, Barcelona.

KaZANGIL, A (1991) «Editorial» en RICS, p. 625.

Lakatos, J. y Musgrave, A (1975) La critica y el desarrollo del conocimiento, Grijalbo, Barcelona. (La edición en castellano de esta obra contiene una interesante introduc- 
ción escrita por J. Muguerza «La teoría de las revoluciones científicas (Una revolución en la teoría contemporánea de la ciencia).

Levi-Strauss, C.; Las estructuras elementales del parentesco, PUF,1949. Anthropologie structurale deux, Plon, París, 1973 Anthropologie structurales, Plon, París, 1958 La pensée sauvage, Plon, París, 1962.

Mendez, R. y Molinero, F. (1991 «Ed.») Espacios y sociedades. Introducción a la Geografía regional del mundo, Ariel Geografía, 1984.

Mumford, L. (1978) Técnica y civilización, Alianza Universidad, Madrid.

Piaget, J. (1974) Le structuralisme, P.U.F., París, 1968. (Existe una traducción al castellano de Oikos-Tau, Barcelona).

PRICE, M. (1989) "Global Change: defining the "ill-defined"», en Environment, 31, 8, octubre, p. 42-44.

Quesada, S. (1978) Metodología y teoría de sistemas: aplicaciones a la Geografía y a las Ciencias Sociales, tesis de licenciatura, Facultad de Geografía e Historia, Barcelona.

Quesada, S. (1978) «La Teoria de los sistemas y la Geografía humana», Geocrítica, n ${ }^{\circ}$ 14, septiembre.

Santos, M. (1990) Por una geografía nueva, Madrid, Espasa Calpe.

SEverino, E (1991) La filosofía futura, Ariel, Barcelona. (Véase especialmente capítulos VIII-XI).

SEverino, E. (1987) La filosofía contemporánea, Ariel, Barcelona.

Simon, H. H. (1957) Models of man. (citado por H. Capel en Filosofia...).

STOdDART, D. R. (1967) «Organism and ecosystem as geographical models» in Models in Geography, Methuen, London, pp. 511-548.

Sweezy, P. M. (1972) Crítica a la ciencia económica, Periferia, Buenos Aires.

Tamames, R. (1974) La polémica sobre los limites del crecimiento, F.C.E., México.

Tuan, Yi-Fu (1976) «Humanistic Geography» Annals of the Association of American Geographers, vol. $66, \mathrm{n}^{\circ} 2$, pp. 266-276 citado por H. Capel en Filosofía...

RESUMEN.-Este trabajo tiene como finalidad aproximarse a la comprensión de los mecanismos básicos que permiten avanzar en el conocimiento del funcionamiento del medio físico y social, así como sus interacciones. El objetivo se lleva a cabo a partir del modelo teórico posibilista, de su enriquecimiento a través de las aportaciones estructuralistas (como la Teoría General de los Sistemas) y de perspectivas teóricas recientes, como las basadas en la Teoría del Cambio Global.

Palabras Clave.-Posibilismo. Estructuralismo. Análisis sistémico. Teoría del Cambio Global. Teoría General de las relaciones hombre-naturaleza.

ABSTRACT.-The purpose of this paper is to gain a better understanding of the basic mechanisms that facilitate advances in knowledge of the working of physical and social environments, as well as their interactions. This objective is reached by starting from the theoretical model of possibilism, of this enrichment through structuralist contributions (such as the General Theory of Systems) and of recent theoretical perspectives, such as those based on the Theory of Global Change.

KEY words.-Possibilism. Structuralism. Systemic analysis. Theory of Global Change. General Theory of Man-Nature Relationships. 